\title{
A determinant formula for congruence zeta functions of maximal real cyclotomic function fields
}

by

\section{DAisuke Shiomi (Nagoya)}

1. Introduction. Let $k$ be a field of rational functions over a finite field $\mathbb{F}_{q}$ with $q$ elements. Fix a generator $T$ of $k$, and let $R=\mathbb{F}_{q}[T]$ be the polynomial subring of $k$. Let $M$ be a monic polynomial in $R$, and $\Lambda_{M}$ be the $M$-torsion of the Carlitz module. The field $k_{M}$ obtained by adding the points of $\Lambda_{M}$ to $k$ is called the $M$ th cyclotomic function field. For the definition of the Carlitz module and basic facts on cyclotomic function fields, see Section 2 below. Let $k_{M}^{+}$be a "maximal real subfield" of $k_{M}$ which is the decomposition field of the infinite prime of $k$ in $k_{M} / k$.

Define $h_{k_{M}^{+}}$to be the order of the divisor class group of degree 0 for $k_{M}^{+}$. Bae and Kang obtained a determinant formula for $h_{k_{M}^{+}}$in [1]. For the field $k_{M}^{+}$, the congruence zeta function $\zeta\left(s, k_{M}^{+}\right)$is expressed by

$$
\zeta\left(s, k_{M}^{+}\right)=\frac{P_{k_{M}^{+}}\left(q^{-s}\right)}{\left(1-q^{-s}\right)\left(1-q^{1-s}\right)}
$$

where $P_{k_{M}^{+}}(X)$ is a polynomial with integral coefficients, and $P_{k_{M}^{+}}(1)=h_{k_{M}^{+}}$ (cf. [5, p. 130]).

The purpose of this paper is to give a determinant formula for $P_{k_{M}^{+}}(X)$ (see Section 3). Since $P_{k_{M}^{+}}(1)=h_{k_{M}^{+}}$, our formula is a generalization of the determinant formula for $h_{k_{M}^{+}}$. As an application, we calculate some low coefficients of $P_{k_{M}^{+}}(X)$ by using the first and second derivatives of a determinant (see Section 4).

2. Basic facts. In this section, we recall some basic properties of cyclotomic function fields and their congruence zeta functions. For details, see $[2,3,4]$.

2000 Mathematics Subject Classification: 11M38, 11R60.

Key words and phrases: congruence zeta function, cyclotomic function fields. 
2.1. Cyclotomic function fields. Let $\operatorname{End}\left(k^{\mathrm{ac}}\right)$ be the $\mathbb{F}_{q^{-}}$-algebra of endomorphisms of the additive group of the algebraic closure $k^{\text {ac }}$ of $k$. The Frobenius automorphism $\varphi\left(x \mapsto x^{q}\right)$ and the $T$-multiplication $\mu_{T}(x \mapsto T \cdot x)$ are elements of $\operatorname{End}\left(k^{\mathrm{ac}}\right)$. We define

$$
x^{M}:=M\left(\varphi+\mu_{T}\right)(x)
$$

for $x \in k^{\text {ac }}$ and $M \in R$. Then $k^{\text {ac }}$ becomes an $R$-module with the above action.

For a monic polynomial $M \in R$, let $\Lambda_{M}$ be the set of all $x$ satisfying $x^{M}=0$, which is a cyclic $R$-submodule of $k^{\text {ac }}$. We have the following isomorphism of $R$-modules:

$$
R / M \rightarrow \Lambda_{M}\left(A \bmod M \mapsto \lambda^{A}\right)
$$

where $\lambda$ is one of the generators of $\Lambda_{M}$.

Let $(R / M)^{\times}$be the group of units of $R / M$. Let $\Phi(M)$ be the order of $(R / M)^{\times}$. By using the previous isomorphism, we see that $\Phi(M)$ is the number of generators of $\Lambda_{M}$.

Let $k_{M}$ be the field obtained by adding the elements of $\Lambda_{M}$ to $k$, which is called the $M$ th cyclotomic function field. Then $k_{M}$ is an abelian extension of $k$. Fix a generator $\lambda$ of $\Lambda_{M}$. We get the following isomorphism:

$$
(R / M)^{\times} \rightarrow \operatorname{Gal}\left(k_{M} / k\right)\left(A \bmod M \mapsto \sigma_{A \bmod M}\right)
$$

where $\operatorname{Gal}\left(k_{M} / k\right)$ is the Galois group of $k_{M} / k$, and $\sigma_{A \bmod M}$ is the isomorphism given by $\sigma_{A \text { mod } M}(\lambda)=\lambda^{A}$. The extension degree of $k_{M} / k$ is $\Phi(M)$. We see that $\mathbb{F}_{q}^{\times}$is contained in $(R / M)^{\times}$, and let $k_{M}^{+}$be the subfield of $k_{M}$ corresponding to $\mathbb{F}_{q}^{\times}$. We call $k_{M}^{+}$the maximal real subfield of $k_{M}$. The extension degree of $k_{M}^{+} / k$ is $\Phi(M) /(q-1)$. If $M$ is a monic polynomial of degree 1 , then $k_{M}^{+}=k$.

For a monic polynomial $M \in R$, let $X_{M}$ be the group of all primitive Dirichlet characters of $(R / M)^{\times}$. We call $\chi$ the real character if $\chi(a)=1$ for any $a \in \mathbb{F}_{q}^{\times}$. Let $X_{M}^{+}$be the set of real characters contained in $X_{M}$. Let $\mathbb{D}$ be the group of all primitive Dirichlet characters. Put

$$
\tilde{k}:=\bigcup_{M} k_{M}
$$

where $M$ runs through all monic polynomials in $R$. By the same argument as in Chapter 3 of [4], we have a one-to-one correspondence between finite subgroups of $\mathbb{D}$ and finite subextension fields of $\tilde{k} / k$, and $X_{M}, X_{M}^{+}$corresponds to $k_{M}, k_{M}^{+}$respectively.

Theorem 2.1 (cf. [4, Theorem 3.7]). Let $X$ be a finite subgroup of $\mathbb{D}$, and $L$ the associated field. For an irreducible monic polynomial $P \in R$, put

$$
Y:=\{\chi \in X \mid \chi(P) \neq 0\}, \quad Z:=\{\chi \in X \mid \chi(P)=1\} .
$$


Then

$$
\begin{aligned}
& X / Y \simeq \text { the inertia group of } P \text { for } L / k, \\
& Y / Z \simeq \text { the cyclic group of order } f_{P}, \\
& X / Z \simeq \text { the decomposition group of } P \text { for } L / k,
\end{aligned}
$$

where $f_{P}$ is the residue class degree of $P$ in $L / k$.

2.2. The congruence zeta function for $k_{M}^{+}$. For a monic polynomial $M \in R$, let $\mathcal{O}_{k_{M}^{+}}$be the integral closure of $R$ in the field $k_{M}^{+}$. We define $\zeta\left(s, \mathcal{O}_{k_{M}^{+}}\right)$by

$$
\zeta\left(s, \mathcal{O}_{k_{M}^{+}}\right):=\prod_{\mathcal{P}}\left(1-\frac{1}{\mathcal{N} \mathcal{P}^{s}}\right)^{-1}
$$

where $\mathcal{P}$ runs through all primes of $\mathcal{O}_{k_{M}^{+}}$, and $\mathcal{N} \mathcal{P}$ denotes the number of elements of the residue field of $\mathcal{P}$. By the same argument as in the case of number fields, we have the following proposition.

Proposition 2.1 (cf. [4, Theorem 4.3]).

$$
\zeta\left(s, \mathcal{O}_{k_{M}^{+}}\right)=\prod_{\chi \in X_{M}^{+}} L(s, \chi)
$$

where the L-function is defined by

$$
L(s, \chi):=\prod_{P}\left(1-\frac{\chi(P)}{\mathcal{N} P^{s}}\right)^{-1}
$$

with $P$ running through all monic irreducible polynomials of $R$.

The congruence zeta function of $k_{M}^{+}$is defined by

$$
\zeta\left(s, k_{M}^{+}\right):=\prod_{\mathcal{P}}\left(1-\frac{1}{\mathcal{N} \mathcal{P}^{s}}\right)^{-1}
$$

where $\mathcal{P}$ runs through all primes of $k_{M}^{+}$. Let $P_{\infty}$ be the infinite prime of $k$ determined by the unique pole of $T$. Let $e_{\infty}, f_{\infty}, g_{\infty}$ be the ramification index in $k_{M}^{+} / k$, the residue class degree, and the number of primes lying above $P_{\infty}$, respectively. Then we obtain

$$
\zeta\left(s, k_{M}^{+}\right)=\zeta\left(s, \mathcal{O}_{k_{M}^{+}}\right)\left(1-q^{-s f_{\infty}}\right)^{-g_{\infty}} .
$$

Since $P_{\infty}$ splits completely in $k_{M}^{+} / k$, we get

$$
\zeta\left(s, k_{M}^{+}\right)=\zeta\left(s, \mathcal{O}_{k_{M}^{+}}\right)\left(1-q^{-s}\right)^{-\Phi(M) /(q-1)} .
$$


3. The determinant formula for $P_{k_{M}^{+}}(X)$. The goal of this section is to give a determinant formula for $P_{k_{M}^{+}}(X)$.

For a monic polynomial $M \in R$ of degree $d(d \geq 2)$, we define $\mathcal{R}_{M}:=$ $(R / M)^{\times} / \mathbb{F}_{q}^{\times}$. For $\alpha \in(R / M)^{\times}$, let $r_{\alpha}$ be the element of $R$ satisfying

$$
r_{\alpha} \equiv \alpha \bmod M, \quad \operatorname{deg} r_{\alpha}<d,
$$

where $\operatorname{deg} A$ denotes the degree of the polynomial $A$. We define

$$
\operatorname{Deg}(\alpha)=\operatorname{deg} r_{\alpha}
$$

We can easily see that Deg is a function over $\mathcal{R}_{M}$.

Let $N=\Phi(M) /(q-1)-1$. We put

$$
\mathcal{R}_{M}=\left\{1, \alpha_{1}, \ldots, \alpha_{N}\right\},
$$

and

$$
\begin{aligned}
d_{i} & =\operatorname{Deg}\left(\alpha_{i}\right) & & (i=1, \ldots, N), \\
d_{i j} & =\operatorname{Deg}\left(\alpha_{i} \alpha_{j}^{-1}\right) & & (i, j=1, \ldots, N) .
\end{aligned}
$$

We define

$$
J_{k_{M}^{+}}(X):=\prod_{\substack{\chi \in X_{M}^{+} \\ \chi \neq 1}} \prod_{Q \mid M}\left(1-\chi(Q) X^{\operatorname{deg} Q}\right),
$$

where $Q$ runs through all irreducible monic polynomials dividing $M$. We put

$$
D_{k_{M}^{+}}(X):=\left(\frac{X^{d_{i j}}-X^{d_{i}}}{1-X}\right)_{i, j=1, \ldots, N} .
$$

Proposition 3.1.

$$
J_{k_{M}^{+}}(X)=\prod_{Q \mid M} \frac{\left(1-X^{f_{Q} \operatorname{deg} Q}\right)^{g_{Q}}}{1-X^{\operatorname{deg} Q}}
$$

where $Q$ is an irreducible monic polynomial dividing $M$ and $f_{Q}, g_{Q}$ are the residue class degree in $k_{M}^{+} / k$ and the number of primes lying over $Q$, respectively.

Proof. Let $Q$ be an irreducible monic polynomial dividing $M$, and put

$$
Y_{Q}^{+}:=\left\{\chi \in X_{M}^{+} \mid \chi(Q) \neq 0\right\}, \quad Z_{Q}^{+}:=\left\{\chi \in X_{M}^{+} \mid \chi(Q)=1\right\} .
$$


From Theorem 2.1,

$$
\begin{aligned}
\prod_{\chi \in X_{M}^{+}}\left(1-\chi(Q) X^{\operatorname{deg} Q}\right) & =\prod_{\chi \in Y_{Q}^{+}}\left(1-\chi(Q) X^{\operatorname{deg} Q}\right) \\
& =\prod_{\chi \in Y_{Q}^{+} / Z_{Q}^{+}} \prod_{\psi \in Z_{Q}^{+}}\left(1-\chi \psi(Q) X^{\operatorname{deg} Q}\right) \\
& =\left(\prod_{\chi \in Y_{Q}^{+} / Z_{Q}^{+}}\left(1-\chi(Q) X^{\operatorname{deg} Q}\right)\right)^{g_{Q}} .
\end{aligned}
$$

Since $Y_{Q}^{+} / Z_{Q}^{+}$is a cyclic group of order $f_{Q}$, we have

$$
\prod_{\chi \in Y_{Q}^{+} / Z_{Q}^{+}}\left(1-\chi(Q) X^{\operatorname{deg} Q}\right)=1-X^{f_{Q} \operatorname{deg} Q} .
$$

Hence we obtain

$$
\prod_{\chi \in X_{M}^{+}}\left(1-\chi(Q) X^{\operatorname{deg} Q}\right)=\left(1-X^{f_{Q} \operatorname{deg} Q}\right)^{g_{Q}} .
$$

From the above equality, the desired result follows.

From Proposition 3.1, $J_{k_{M}^{+}}(X)$ is a polynomial with integral coefficients. Now we can prove the main result of the present paper.

THEOREM 3.1. Let $M \in R$ be a monic polynomial of degree not less than 2. Then

$$
\operatorname{det} D_{k_{M}^{+}}(X)=P_{k_{M}^{+}}(X) J_{k_{M}^{+}}(X) .
$$

Proof. For any $\chi \in X_{M}^{+}$, let $f_{\chi}$ be the conductor of $\chi$. Define $\tilde{\chi}$ by

$$
\tilde{\chi}=\chi \circ \pi_{\chi}
$$

where $\pi_{\chi}:(R / M)^{\times} \rightarrow\left(R / f_{\chi}\right)^{\times}$is the natural homomorphism. Then we can easily see that

$$
L(s, \tilde{\chi})=L(s, \chi) \cdot \prod_{Q \mid M}\left(1-\chi(Q) q^{-s \operatorname{deg} Q}\right) .
$$

Hence we have

$$
\prod_{\substack{\chi \in X_{M}^{+} \\ \chi \neq 1}} L(s, \tilde{\chi})=\left(\prod_{\substack{\chi \in X_{M}^{+} \\ \chi \neq 1}} L(s, \chi)\right) \cdot J_{k_{M}^{+}}\left(q^{-s}\right)=\zeta\left(s, \mathcal{O}_{k_{M}^{+}}\right)\left(1-q^{1-s}\right) J_{k_{M}^{+}}\left(q^{-s}\right) .
$$

By the same argument as in Lemma 3 in [2], if $\chi \neq 1$,

$$
L(s, \tilde{\chi})=\sum_{k=0}^{d-1} \sum_{\substack{\operatorname{deg} A=k \\ A \text { monic }}} \tilde{\chi}(A) q^{-k s}=\sum_{\alpha \in \mathcal{R}_{M}} \tilde{\chi}(\alpha) q^{-\operatorname{Deg}(\alpha) s} .
$$


Since $\tilde{\chi}$ is real, $\tilde{\chi}$ is a character of $\mathcal{R}_{M}$. Notice that $\tilde{\chi}$ runs through all characters of $\mathcal{R}_{M}$ when $\chi$ runs through all characters of $X_{M}^{+}$. By the Frobenius determinant formula (cf. [4, Lemma 5.26]),

$$
\prod_{\substack{\chi \in X_{M}^{+} \\ \chi \neq 1}} L(s, \tilde{\chi})=\prod_{\substack{\chi \in X_{M}^{+} \\ \chi \neq 1}} \sum_{\alpha \in \mathcal{R}_{M}} \tilde{\chi}(\alpha) q^{-\operatorname{Deg}(\alpha) s}=\operatorname{det}\left(q^{-s d_{i j}}-q^{-s d_{i}}\right)_{i, j=1, \ldots, N} .
$$

Since $P_{\infty}$ splits completely in $k_{M}^{+} / k$, we have

$$
\operatorname{det}\left(\frac{q^{-s d_{i j}}-q^{-s d_{i}}}{1-q^{-s}}\right)_{i, j}=P_{k_{M}^{+}}\left(q^{-s}\right) J_{k_{M}^{+}}\left(q^{-s}\right) .
$$

Putting $X=q^{-s}$, we obtain the desired result.

By applying L'Hôpital's rule, we calculate

$$
\left.\frac{X^{d_{i j}}-X^{d_{i}}}{1-X}\right|_{X=1}=d_{i}-d_{i j}
$$

We can now use our theorem to rederive the class number formula of Bae and Kang.

Corollary 3.1 (Bae-Kang [1]). In the notations of Proposition 3.1, we have

$$
\operatorname{det}\left(d_{i}-d_{i j}\right)_{i, j=1, \ldots, N}=W_{k_{M}^{+}} h_{k_{M}^{+}}
$$

where

$$
W_{k_{M}^{+}}= \begin{cases}\prod_{Q \mid M} f_{Q} & \text { if } g_{Q}=1 \text { for every prime } Q \text { dividing } M \\ 0 & \text { otherwise }\end{cases}
$$

Proof. We can calculate

$$
\left.\operatorname{det} D_{k_{M}^{+}}(X)\right|_{X=1}=\operatorname{det}\left(d_{i}-d_{i j}\right)_{i, j=1, \ldots, N},
$$

and $W_{K_{M}^{+}}=J_{M}^{+}(1)$ by Proposition 3.1. Since $P_{k_{M}^{+}}(1)=h_{k_{M}^{+}}$, we obtain the desired result.

REMARK. The corollary applies, in particular, when $M=Q^{d}$ is a prime power. Since $Q$ is totally ramified in $k_{M}^{+} / k$, we have $g_{Q}=1$ and $f_{Q}=1$. It follows, in this case, that $h_{k_{M}^{+}}=\operatorname{det}\left(d_{i}-d_{i j}\right)$.

Corollary 3.2. Let $M \in R$ be a monic polynomial of degree 2. Then $P_{k_{M}^{+}}(X)=1$.

Proof. We have

$$
d_{i}=1, \quad d_{i j}= \begin{cases}0 & \text { if } i=j, \\ 1 & \text { if } i \neq j .\end{cases}
$$

It follows that $D_{k_{M}^{+}}(X)=I_{N}$. By Theorem 3.1, $P_{k_{M}^{+}}(X)=1$. 
I would like to thank the referee for suggesting the following alternative proof of Corollary 3.2. Using the Riemann-Hurwitz formula, we find that $k_{M}^{+}$ has genus zero in the case of $\operatorname{deg} M=2$. Thus, we also obtain $P_{K_{M}^{+}}(X)=1$.

We give some examples of $P_{k_{M}^{+}}(X)$.

EXAmple 3.1. Let $q=2$ and $M=T^{3} \in \mathbb{F}_{q}[T]$. We put

$$
\mathcal{R}_{M}=\left\{1, \alpha_{1}=T+1, \alpha_{2}=T^{2}+1, \alpha_{3}=T^{2}+T+1\right\} .
$$

As $M$ is a power of an irreducible polynomial, $P_{k_{M}^{+}}(X)=\operatorname{det} D_{k_{M}^{+}}(X)$. Hence

$$
P_{k_{M}^{+}}(X)=\operatorname{det} D_{k_{M}^{+}}(X)=\left|\begin{array}{rrr}
1 & -X & -X \\
X & 1+X & 0 \\
0 & X & 1+X
\end{array}\right|=1+2 X+2 X^{2} .
$$

EXAmPle 3.2. Let $q=2$ and $M=T^{2}(T+1)^{2} \in \mathbb{F}_{q}[T]$. We put

$$
\mathcal{R}_{M}=\left\{1, \alpha_{1}=T^{2}+T+1, \alpha_{2}=T^{3}+T+1, \alpha_{3}=T^{3}+T^{2}+1\right\} .
$$

Then

$$
\begin{aligned}
\operatorname{det} D_{k_{M}^{+}}(X) & =\left|\begin{array}{rrr}
1+X & -X^{2} & -X^{2} \\
0 & 1+X+X^{2} & X^{2} \\
0 & X^{2} & 1+X+X^{2}
\end{array}\right| \\
& =\left(1+X+2 X^{2}\right)(1+X)^{2},
\end{aligned}
$$

and

$$
J_{k_{M}^{+}}(X)=(1+X)^{2}
$$

Thus, we get

$$
P_{k_{M}^{+}}(X)=1+X+2 X^{2}
$$

4. Calculating the coefficients of $\operatorname{det} D_{k_{M}^{+}}(X)$. In this section, we will give a formula for the coefficients of low degree for $\operatorname{det} D_{k_{M}^{+}}(X)$.

Let $M \in R$ be a monic polynomial of degree $d$. Since $\operatorname{det} D_{k_{M}^{+}}(0)=1$, we can write

$$
\operatorname{det} D_{k_{M}^{+}}(X)=1+a_{1} X+a_{2} X^{2}+\cdots,
$$

where $a_{i}(i=1,2, \ldots)$ are integers. For $0 \leq i<d$, put

$$
s_{i}=\#\left\{\alpha \in \mathcal{R}_{M} \mid \operatorname{deg} \alpha=i\right\}, \quad t_{i}=\#\left\{\alpha \in \mathcal{R}_{M} \mid \operatorname{deg} \alpha \leq i\right\},
$$

where $\# A$ is the number of elements of the set $A$. We have the following result. 
Proposition 4.1. If $\operatorname{deg} M \geq 3$, then

$$
\begin{aligned}
& a_{1}=\frac{\Phi(M)}{q-1}-t_{1}, \\
& a_{2}=\frac{1}{2}\left\{\frac{\Phi(M)}{q-1}-2 t_{2}+\left(\frac{\Phi(M)}{q-1}-t_{1}\right)^{2}+t_{1}^{2}\right\} .
\end{aligned}
$$

To prove this proposition, we first state the following lemma, which can be shown by simple calculations.

LEMMA 4.1. Let $F(X)=\left(f_{i j}(X)\right)_{i, j}$ be a matrix-valued function of one variable. If $F(X)$ is twice differentiable and invertible for $X=X_{0}$, then

$$
\begin{aligned}
\left.\frac{d \operatorname{det} F(X)}{d X}\right|_{X=X_{0}}= & \operatorname{det} F\left(X_{0}\right) \cdot \operatorname{Tr}\left(F\left(X_{0}\right)^{-1} \frac{d F}{d X}\left(X_{0}\right)\right) \\
\left.\frac{d^{2} \operatorname{det} F(X)}{d X^{2}}\right|_{X=X_{0}}= & \operatorname{det} F\left(X_{0}\right) \cdot\left\{\operatorname{Tr}\left(F\left(X_{0}\right)^{-1} \frac{d^{2} F}{d X^{2}}\left(X_{0}\right)\right)\right. \\
& -\operatorname{Tr}\left(F\left(X_{0}\right)^{-1} \frac{d F}{d X}\left(X_{0}\right) F\left(X_{0}\right)^{-1} \frac{d F}{d X}\left(X_{0}\right)\right) \\
& \left.+\operatorname{Tr}\left(F\left(X_{0}\right)^{-1} \frac{d F}{d X}\left(X_{0}\right)\right)^{2}\right\}
\end{aligned}
$$

where $\operatorname{Tr}(A)$ is the trace of the matrix $A$.

Proof of Proposition 4.1. The matrix $D_{k_{M}^{+}}(0)$ is the unit matrix $I_{N}$, and

$$
D_{k_{M}^{+}}(0)^{-1}=I_{N}, \quad \frac{d D_{k_{M}^{+}}}{d X}(0)=\left(c_{i j}\right)_{i, j=1, \ldots, N},
$$

where

$$
c_{i j}= \begin{cases}0 & \text { if } i=j, d_{i}=1 \\ 1 & \text { if } i=j, d_{i} \neq 1 \\ 1 & \text { if } d_{i j}=1, d_{i}>1 \\ -1 & \text { if } d_{i j}>1, d_{i}=1 \\ 0 & \text { otherwise }\end{cases}
$$

From Lemma 4.1, we obtain

$$
a_{1}=\operatorname{Tr}\left(\left(c_{i j}\right)_{i, j}\right)=\frac{\Phi(M)}{q-1}-t_{1}
$$

and

$$
2 a_{2}=\operatorname{Tr}\left(\frac{d^{2} D_{k_{M}^{+}}}{d X^{2}}(0)\right)-\operatorname{Tr}\left(\left(\frac{d D_{k_{M}^{+}}}{d X}(0)\right)^{2}\right)+\operatorname{Tr}\left(\frac{d D_{k_{M}^{+}}}{d X}(0)\right)^{2} .
$$


By straightforward calculations, we get

$$
\operatorname{Tr}\left(\frac{d^{2} D_{k_{M}^{+}}}{d X^{2}}(0)\right)=2\left(\frac{\Phi(M)}{q-1}-t_{2}\right), \quad \operatorname{Tr}\left(\frac{d D_{k_{M}^{+}}}{d X}(0)\right)^{2}=\left(\frac{\Phi(M)}{q-1}-t_{1}\right)^{2} .
$$

From (21),

$$
\begin{aligned}
\operatorname{Tr}\left(\left(\frac{d D_{k_{M}^{+}}}{d X}(0)\right)^{2}\right) & =\sum_{i=1}^{N} \sum_{j=1}^{N} c_{i j} c_{j i} \\
& =\sum_{i=1}^{N} c_{i}^{2}+\sum_{\substack{d_{i}=1<d_{i j} \\
d_{j}=1<d_{j i}}} 1+\sum_{\substack{d_{i j}=1<d_{i} \\
d_{j i}=1<d_{j}}} 1-\sum_{\substack{d_{i}=1<d_{i j} \\
d_{j i}=1<d_{j}}} 1-\sum_{\substack{d_{j}=1<d_{j i} \\
d_{i j}=1<d_{i}}} 1 .
\end{aligned}
$$

Since $\operatorname{deg} M \geq 3$, we can easily see that

$$
\begin{gathered}
\sum_{i=1}^{N} c_{i}^{2}=\frac{\Phi(M)}{q-1}-t_{1}, \quad \sum_{\substack{d_{i}=1<d_{i j} \\
d_{j}=1<d_{j i}}} 1=s_{1}^{2}-s_{1}, \\
\sum_{\substack{d_{i j}=1<d_{i} \\
d_{j i}=1<d_{j}}} 1=0, \quad \sum_{\substack{d_{i}=1<d_{i j} \\
d_{j i}=1<d_{j}}} 1=\sum_{\substack{d_{j}=1<d_{j i} \\
d_{i j}=1<d_{i}}} 1=s_{1}^{2} .
\end{gathered}
$$

It follows that

$$
\operatorname{Tr}\left(\left(\frac{d D_{k_{M}^{+}}}{d X}(0)\right)^{2}\right)=\frac{\Phi(M)}{q-1}-t_{1}^{2} .
$$

Hence (20) follows.

We give some examples for Proposition 4.1.

EXAMPle 4.1. Let $M \in R$ be an irreducible monic polynomial of degree 3 . Then

$$
t_{1}=q+1, \quad t_{2}=\frac{\Phi(M)}{q-1}=q^{2}+q+1 .
$$

By Proposition 4.1,

$$
P_{k_{M}^{+}}(X)=\operatorname{det} D_{k_{M}^{+}}(X)=1+q^{2} X+\frac{q\left(q^{3}+1\right)}{2} X^{2}+\cdots .
$$

EXAmple 4.2. We put $M=T^{n}(n \geq 3)$. Then

$$
t_{1}=q, \quad t_{2}=q^{2}, \quad \frac{\Phi(M)}{q-1}=q^{n-1} .
$$

Hence

$$
\begin{aligned}
P_{k_{M}^{+}}(X) & =\operatorname{det} D_{k_{M}^{+}}(X) \\
& =1+\left(q^{n-1}-q\right) X+\frac{q^{n-1}\left(q^{n-1}-2 q+1\right)}{2} X^{2}+\cdots .
\end{aligned}
$$


Acknowledgements. I would like to thank Prof. Kohji Matsumoto and the referee for their valuable comments.

\section{References}

[1] S. H. Bae and P.-L. Kang, Class numbers of cyclotomic function fields, Acta Arith. 102 (2002), 251-259.

[2] S. Galovich and M. Rosen, The class number of cyclotomic function fields, J. Number Theory 13 (1981), 363-375.

[3] D. R. Hayes, Explicit class field theory for rational function fields, Trans. Amer. Math. Soc. 189 (1974), 77-91.

[4] L. C. Washington, Introduction to Cyclotomic Fields, Springer, New York, 1982.

[5] A. Weil, Basic Number Theory, Springer, New York, 1967.

Graduate School of Mathematics

Nagoya University

Chikusa-ku, Nagoya 464-8602

Japan

E-mail: m05019e@math.nagoya-u.ac.jp

Received on 18.4.2008

and in revised form on 10.3.2009 